\title{
EVALUATION OF A SOCIAL MULTIPLAYER GAME FEATURING MULTIMODAL INTERACTION
}

\author{
Alf Inge Wang ${ }^{1}$, Esben André Føllesdal ${ }^{2}$ \\ Dept. of Computer and Information Science, Norwegian University of Science and Technology \\ Sem Sælandsv. 7-9, N-7491 Trondheim, Norway \\ 1)alfw@idi.ntnu.no / 2)esbenaf@idi.ntnu.no
}

\begin{abstract}
In this paper, we describe and evaluate a new multiplayer gaming platform, which combines the strength of mobile gaming, PC gaming and large screens. Mobile phones are used as game controllers that also provide the player with an individual screen for status information, score, game choices etc. By using a mobile phone as a game controller, it is possible for to give individual feedback to the player such as sound, vibration, and graphics. The game itself is played via a PC server that displays the game on a large screen. This gives a unique social in-thesame-room gaming experience. The paper describes an evaluation of the gaming platform as well as a specific game, SelFish, where the focus of the evaluation was on identifying strength and weaknesses of the game and the platform related to some design recommendations. The evaluation was performed as a user test where 91 subjects participated by playing a game, and then filling out a questionnaire. The results reveal some design flaws, issues related to the use a mobile phone as a game controller, and some social effects of the game.
\end{abstract}

\section{KEY WORDS}

Multimedia Systems, Mobile and Wireless Computing, Software Evaluation, Graphical User Interfaces, and Games.

\section{Introduction}

Mobile phones have now become much more than a simple communication tool we can use to talk to each other, as they have made significant progress in areas like network connectivity, CPU power, size of memory, integrated hardware (such as sensors, GPS, camera, audio recording and playback, graphic chips, high resolution displays), and more. In addition, mobile phones come with advanced operating systems that share many of the capabilities of operating systems for PCs. It is therefore essential to explore new usage areas of mobile phones beyond the interactive systems that only run on the mobile device. "The mobile phone is enabling new ways of engaging with media content, and new ways of negotiating and communicating within both actual and virtual environments" [1]. Although mobile gaming has become popular, gaming on mobile phones gives an inferior gaming experience compared $\mathrm{PC}$ and console games due to a very small screen and a less immersive multiplayer experience. In our research we wanted to overcome these shortcomings by developing a new gaming platform.

In the MOOSES (Multiplayer On One Screen Entertainment System) project, we wanted to combine the strengths of mobile gaming with the strengths of PC gaming to provide a new gaming platform. Further, we wanted this new gaming platform to focus on the in-the-same-room social aspects of gaming, which is very different from multiplayer online games since the players can directly talk and interact with each other physically when playing the same game. Our approach is a mixture of the dual screen feature of handheld game console Nintendo DS, interactive movies, and traditional multiplayer games. MOOSES is a framework for developing games played on large screens such as in movie theatres where each player use his own mobile phone as a game controller. Similar to the dual screen approach of Nintendo DS, the screen on the mobile phone is used as a personal screen where the user can get his score, status information, weapon selection, strategic choices etc. The large screen is the game canvas shared by all players where the real action is going on.

This paper describes a specific MOOSES game, SelFish, and an evaluation of this game related to some design recommendations. The evaluation was performed as a user study of 91 subjects that played the game and then filled out a questionnaire. The goal of the user study was to discover strength and weaknesses with the SelFish game specifically and the MOOSES game platform in general and then suggest improvements for the game and the gaming platform.

The rest of the paper is organized as follows: Section 2 describes related work, Section 3 describes to Multiplayer One On Screen Entertainment System, Section 4 presents the game being evaluated in this 
paper, Section 5 describes the evaluation of MOOSES and the game SelFish, and finally Section 6 concludes the paper.

\section{Related Work}

As far as we know, there have not been any attempts to establish a gaming platform such as MOOSES that combines mobile phones with a large screen as we do. However, there are some similar prototypes and there are related approaches of using public large screens, which are described in this section.

In [2], Maynes-Aminzade et. al describe three techniques for interactive audience participation. Their techniques allow audiences of hundreds of people to control onscreen activity by leaning left and right in their seats, batting a beach ball while its shadow is used as a pointing device, and pointing laser pointers at the screen. A video-streaming camera is used to recognize audience movement in their seats, the movement of the shadow of the ball on the screen, and the positions of the light dots from the laser pointers on the screen respectively. The first two techniques (seats and ball) are different from how games are normally designed in MOOSES, as the players are collaboratively controlling the same game object in the game. However, we have also designed games in MOOSES that uses the same technique. The last technique (laser pen) allows each player to interact individually, but the player will not get any individual feedback. Also the individual control is indirect, as laser dots are used as interacting game objects. The paper does not say anything about latency (how immediate the game responses to player events) of using image recognition for controlling game objects, but this is likely to be a problem with this approach.

In [3], Vajk et. al describe how the 3D motion sensor in Nokia 5500 mobile phone can be used as a controller for a multiplayer game displayed on a large screen. The game they developed was a 2D driving game similar to Atari's arcade game Super Sprint from 1986, apart from that in this game the players had to tilt their mobile phones in the direction they wanted to move their car on the large screen. The game was implemented in XNA, and Bluetooth was used for the communication between the $\mathrm{PC}$ and the mobile phones. This paper illustrates that various capabilities of the mobile phones such as sensors can be utilized in MOOSES games. Unlike MOOSES, this was just a prototype of one specific game, the game was not designed to allow many simultaneous players (such as 10 or more), and dynamically entering and leaving the game was not supported.

In [4], Borriello et. al describe experiences from developing a multiplayer video game on a large screen in a "Software for Embedded Systems" class. Each student in the class had to design his own accelerometer-based game controller that was used to allow up to 28 players play a soccer game simultaneously. The motivation of this work was to motivate students to learn design of embedded systems. This work is similar to the MOOSES project, but the focus is very different. In the MOOSES project the focus has been to develop a gaming platform, while for this project it has been to motivate students to create their own game controllers.

Boring et. al describe a similar approach where the accelerometer in a mobile phone was used to interact with content on large public displays [5]. In this paper, the mobile phone is simply used to control a pointer on the large screen, and the approach is evaluated to identify strengths and weaknesses.

In [6], Scheible et. al present the MobiToss application, which is an application for creating and sharing multimedia art on large public displays using mobile phones. First, the user needs to take a photo or capture a video on their mobile phone, and then he can use a "throwing" gesture to transfer the clip onto the large public display. The user can then manipulating his "art" by tilting the phone in different directions.

In [7], Shirazi et. al describe how mobile phones' camera flashlights can be used to interact with a large screen. A camera is placed in front of a large screen facing the users used to track the lights emitted from the users' mobile phones. This technique is mainly used as a way of controlling mouse pointer position, making selections, scrolling/zooming.

Before the screening of the movie Ratatouille in the UK, Volvo had an interactive commercial - also called a game $[8,9]$. In the game, the audience at the movie theatre tried to drive a Volvo through an objects course. Some objects had to be collected and gave extra points, while other object should be avoided. The people at the theatre used their hands to control the game, and by waving both hands to the direction they wanted the car to turn. The fun part of this game was that the people collaboratively controlled the same car. A camera registered the movement of the people in the theatre and the direction of the car was changed corresponding to the majority of the people. 12 movie theatres in UK competed against each other to get the highest score. 
The Ghost in the Cave game aims at players between 13 and 16 years old [10]. The players are divided into two teams where the players on each team have to cooperate to archive the main goal: be the first to enter the third cave. Both teams start in one cave, and must first find the exit of their own cave before they can find the third cave. Each team has one large screen each. The cooperation is based on non-verbal communication, and thus it is possible for players with different cultural background and language to cooperate. Each team chooses one leader. The leader will control the avatar's movement direction on the screen, while the rest of the team controls the avatars speed. The crowd controls the avatar's speed by singing and dancing. Singing loud or dancing fast makes the ghost move quickly and singing low or dancing slow makes the movement slow.

Newsbreaker Live is another cinema style game where the audience controls the game [11]. This is a clone of the 1986 arcade game Arkanoid, where the players try to knock down a wall of bricks. Instead of controlling the pad with the mouse or a joystick, the movement of the crow decides how the pad will go.

Lecture Quiz is a multiplayer quiz game where the students use their mobile phones to answer questions shown on a large screen using the teachers PC $[12,13]$. The main goal of developing this game was to increase the participation and motivation of the students in lectures. Lecture Quiz is very similar to MOOSES, but is limited to quiz games.

The MOOSES project was not based on any of the projects described above when it was initiated. The initial idea was to utilize the large screen in movie theaters for social gaming purposes. However, some of the ideas described in the projects above have been tried out in MOOSES, such as collaborative control of game objects.

\section{Multiplayer On One Screen Entertainment System - MOOSES}

Multiplayer On One Screen Entertainment System (MOOSES) is a framework that was invented and developed at the Norwegian University of Science and Technology (NTNU) in 2006 together with the Norwegian telecom company Tellu. The idea of MOOSES is to provide a multiplayer video game platform where the game is played on a large screen (e.g. in a movie theatre), and the players use their own mobile phones as game controllers. The game platform can be used to replace passive commercials before the movie starts, to attract people to e.g. shopping centers, or just to boost social happenings.

Figure 1 shows an overview of the MOOSES architecture. The MOOSES framework consists of a MOOSES server that handles the communication between the server and the client, a player manager, a game manager, and a payment manager. The MOOSES server communicates via wireless networks with mobile phones running the MOOSES client using a defined MOOSES protocol.

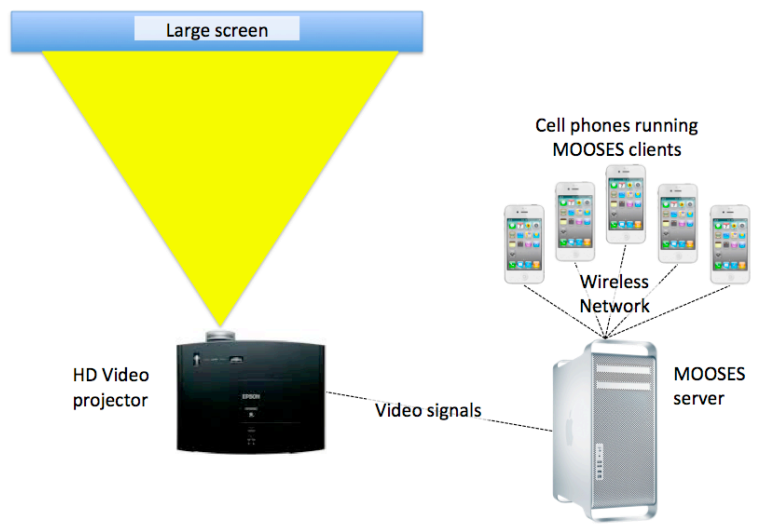

Figure 1. MOOSES overall architecture

Before a player can play a MOOSES game, he must download the MOOSES client to his mobile phone. The MOOSES client can be used to run all MOOSES games available. The MOOSES games themselves are run from the MOOSES server and displayed on a large screen. The MOOSES clients send various controller events to the MOOSES server such as key events, accelerometer changes, video events, audio events etc. The MOOSES server can send back events and data to the MOOSES client that can cause the client to change GUI, play a sound, vibrate, etc. The uniqueness of the MOOSES framework is that the player has two screens available. One personal screen on a mobile phone that can be used for personal game information such as personal score, selection of weapon, personal messages, strategic choices etc, and one shared large screen where all players share the same game canvas. The communication between the clients and the server can go over Bluetooth, WiFi, GPRS, $3 \mathrm{G}$ or other wireless networks. MOOSES has been tested with over 50 players over Bluetooth with only minor latency (about 0.2 seconds) using several Bluetooth hubs to facilitate the communication between server and clients. To improve network performance, different wireless networks can be utilized at the same time. 
The MOOSES games can be developed on many different development platforms. Currently, we have MOOSES games developed in Java, $\mathrm{C}++, \mathrm{XNA}$ and Flash. To play a MOOSES game, the players must start the MOOSES client on their mobile phones (just like starting a game or any other mobile application), and then register a nickname. Then the players get a chance to vote on what game to play, and the game with most votes will be launched after a timer runs out. The game sessions in MOOSES typically lasts from one to five minutes, and players can connect and disconnect during gameplay. After the game session has ended, a high score list will be shown including pictures of the players or their avatars if available.

In the MOOSES project, we identified some design recommendations the game developer should follow to develop a successful MOOSES game:

- The game must be designed and balanced to allow varying numbers of players. The maximum number of players to be allowed in the game depends on the gameplay of the game, but the games should be designed to allow at least ten simultaneous players.

- The game must be designed to allow players to come and go during the gameplay. MOOSES games are social games with short game sessions characterized by people joining and leaving game sessions all the time. This means that the game must be able to dynamically add playable characters to the screen without interfering with the rest of the game, as well to have game mechanics that allows the number of players to change during a game session.

- The game must be designed in such a way that the players can find and identify their own playable character. Usually, playable characters or game objects in MOOSES games have attached a nickname to distinguish between the players. In addition, it is wise to allow the players to choose among different types of looks of their characters before they join a game session. Simply to choose a color is usually not enough to distinguish between players, as it is hard to have more ten distinguishing colors.

- The game concept for MOOSES games needs to be very easy to learn and understand. As the MOOSES game sessions are short, it is important that the players understand what they should do in the game immediately.

- The control of the game must be very simple. As a mobile phone is used as a game controller, it is essential that the control scheme for a game is very simple. In practice, this means that few keys should be used to control the game. Our recommendation is not to have more complicated game controls than directional control and one additional action button.

- The MOOSES game canvas should not be too crowded. The maximum number of players is limited by the required space each player needs to move around and that the playable characters are big enough to immerse the player.

- The MOOSES game concept must be social. The whole idea of the MOOSES platform is to provide an in-the-same-room social gaming platform and the games must reflect this.

The design recommendations listed above are among the things we evaluated in this paper, and the results are described in Section 5.

\section{SelFish - a MOOSES Game}

The concept of the SelFish game was developed by the first author and implemented by the second author of this paper. Figure 2 shows a screenshot of the SelFish game in action.

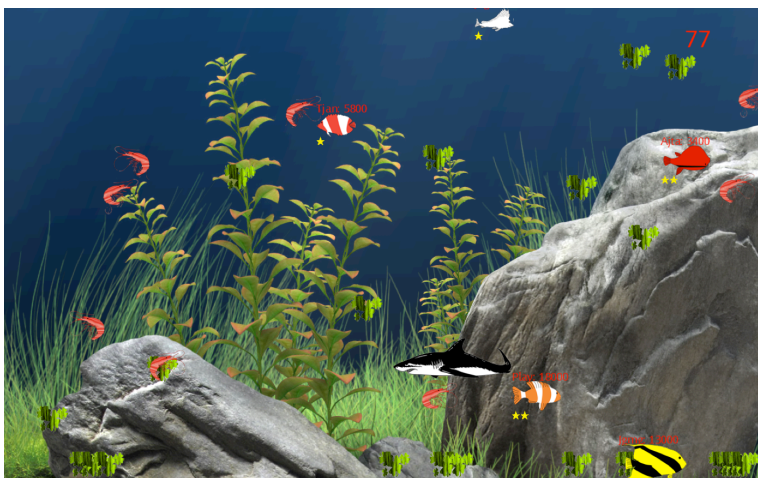

Figure 2. Screenshot from the SelFish game

The game itself was specifically designed to be MOOSES game from ground up, as it supported variation in number of players, allow players to come and go, provide gameplay appropriate for short game sessions, and is competitive and social. SelFish is a game where the player controls a fish that can eat smaller creatures to grow, and he must also avoid getting eaten by larger fishes. All players start with a fish at level 1 that only can eat plankton. When a fish reaches level 2, it can eat shrimps. From level 3 and up to 5 (the highest level), the player's fish can start to eat other players' fishes. For players at the highest level (level 5), it is only computer-controlled sharks that can eat their fishes. If a player's fish has been eaten, it will be respawn (re-appear) after some time as a fish at level 1. Points are given based on how 
much the player's fish eat and what it eats. The game ends when the time is up, and the players are ranked according to their score in the high-score list. The controls in the game are kept simple. The player can control the direction of the fish using the joystick or the $2-4-6-8$ keys on the keypad on the mobile phone. In addition, one extra key is used for speed boost. This features is typically used when the player want to escape from a bigger fish. The speed boost must be recharged before it can be used again. The SelFish game was implemented using Microsoft's game development platform XNA [14].

\section{Evaluating MOOSES and SelFish}

The evaluation of the MOOSES game platform and the SelFish game was performed by in total 91 subjects playing the games in three events: Kreator$0919^{\text {th }}$ March 2009 in Trondheim Norway, Åsane culture center $2^{\text {nd }}$ April in Bergen Norway, and Errors LAN-party $11^{\text {th }}$ of April 2009 in Bodø Norway. Figure 3 shows a picture from a game session at the Errors LAN-party. The user test consisted of game sessions where five to ten players played SelFish simultaneously, followed by a session where the subjects filled out questionnaires.

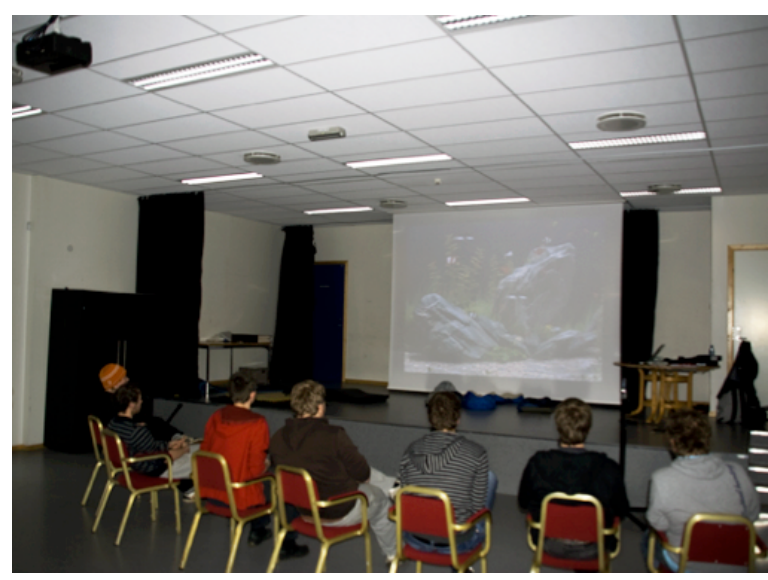

Figure 3. Picture from playing SelFish at Errors LAN-party in Bodø Norway $11^{\text {th }}$ of April 2009

\subsection{Results from the User Tests}

The questionnaire consisted of three parts where the subjects used the Likert's 5-point scale on the two last parts to state the level of agreement to 15 statements using the scores (5) strongly agree, (4) agree, (3) neither, (2) disagree, and (1) strongly disagree.

In the first part of the questionnaire, the subjects stated their sex, age, and time spent on computer games each week. $29 \%$ of the players were female and $71 \%$ male. The age distribution of the players were $11 \% 16$ years or younger, $52 \%$ between 17 and 22 years, and $37 \%$ above 23 . In average, the subjects play 4.43 hours of computer games a week, where the minimum is 0 and the maximum is 60 hours. In this paper we have classified subjects that play 0-2 hours per week as casual players, 3-9 as medium players, and more than 10 hours per week as hardcore gamers.

The second part of the questionnaire consisted of statements directly related to the SelFish game and the usability of this game. The results of the statements related to the SelFish game is shown in Figure 4, where the first part of the bar (from the left) is strongly agree (blue), then agree (red), then neither (green), disagree (purple), and strongly disagree (cyan).

The results in Figure 4 show that $85 \%$ (strongly agree/agree) of the subjects thought that Selfish was a fun game, but only $37 \%$ thought it was an addictive game. $91 \%$ of the subjects understood the goal of the game and $75 \%$ recognized the level of their fish. However, $60 \%$ thought it was difficult to know when the speed-boost was ready, and 39\% had difficulties locating their own fish. If we look at demographical differences in locating their own fish, we find that female subjects on average to a larger degree (2.96) had more problem locating their own fish compared to male subjects (3.26). If we look at differences in age, we find that the youngest players (up to 16 years old) had less problem locating their own fish (3.30) than the oldest players of 23 years or above (3.00). If we consider the hours the subjects play every week, we find that the casual players had an average of 3.15, the medium players had an average of 3.47 , and the hardcore players had an average of 2.94. It is rather strange that the hardcore gamers indicate that they to a larger degree had problems locating their own fish. One possible reason to this could be that they expect more from a game and found this so irritating that they gave it a low score. Regarding the game concept, $88 \%$ of the subjects stated that the SelFish game was suitable regardless of age and sex.

From these results, we can see that the SelFish game is a game suited for the MOOSES game platform but the results also reveal some usability issues. It is important that MOOSES games are fun, easy to understand the goal of the game and that it is suitable regardless of age and sex. The two main usability issues identified are issues typical for the MOOSES format: 1) Hard to identify own playable character on a large screen, and 2) Cumbersome to change focus between the large screen and the screen on the mobile phone (to check if the speed-boost is ready in SelFish). To overcome the first problem, the 
playable character on the screen should be introduced one at a time where the character if first shown large with associate nickname, and then zoomed out to appropriate size and placement on screen. To overcome the second problem, an alternative design could be to use the sound or vibration of the mobile phone to indicate when the speed-boost is ready.

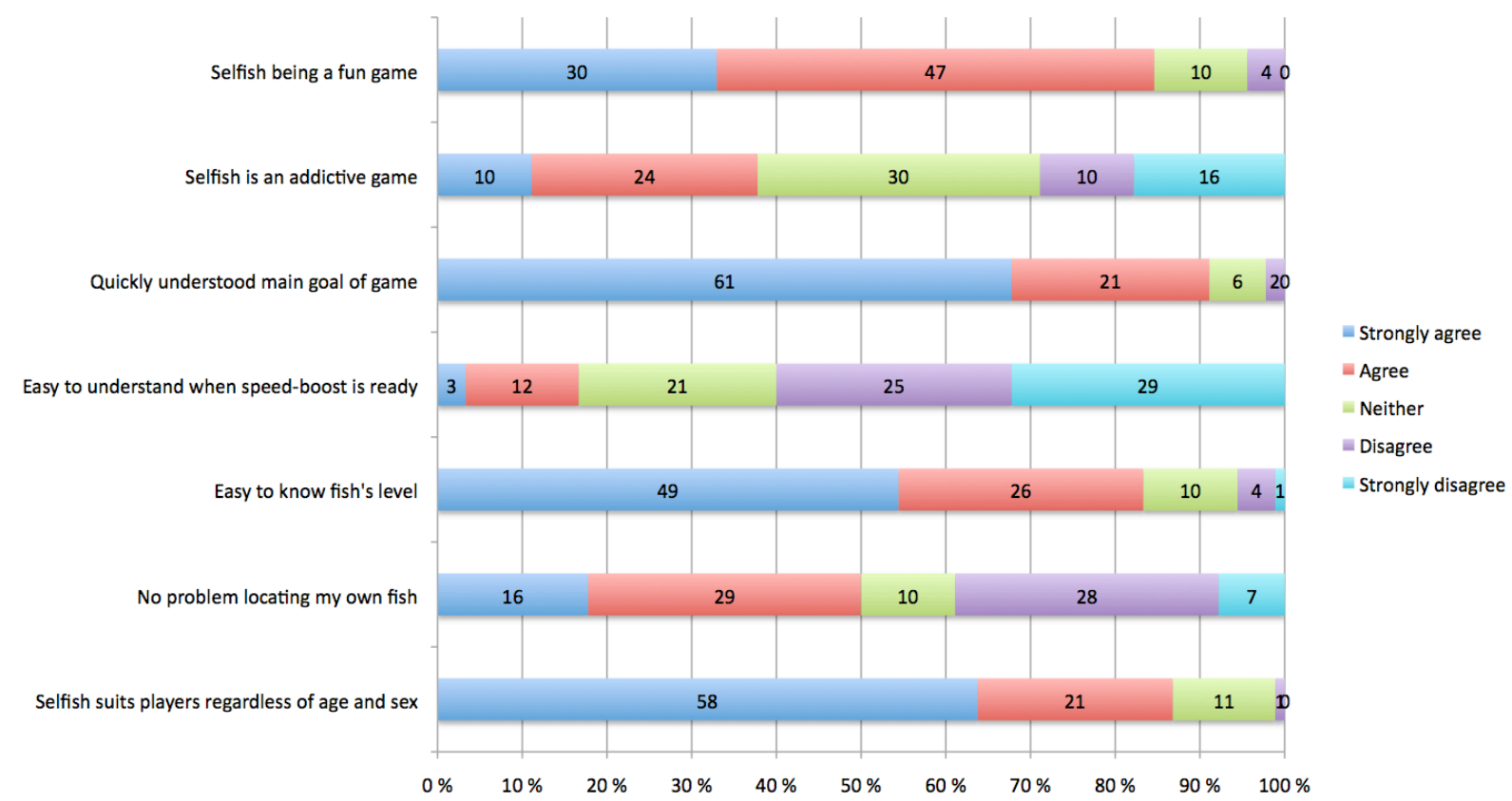

Figure 4. Results from questionnaire on the SelFish game

The third part the questionnaire was related to statements regarding the MOOSES game platform in general with focus on the combination of using mobile phones as controllers, large shared displays, the social experience of such games, connectivity issues, uniqueness of the game platform, and whether users would prefer such games to watching commercials before the movie starts in cinemas.

Figure 5 shows the results from part three in the survey and that the majority of the subjects $(59 \%$ strongly agree or agree, and $23 \%$ neutral) thought that it was easy to use a mobile phone to control a game. Still $18 \%$ thought it was not easy to control the game using a mobile phone. If we look at the demographics, we found female subjects on average agreed more (3.85) compared to male subjects (3.55) on that it was easy to use the mobile phone to control the game. If we look at the age, the differences are much more visible. For subjects between 10-16, the mean value was 4.00 , between 17-22 the mean value was 3.57 , and for subjects above 22 the mean value was 2.48. This means that the average for the youngest players is (4) agree, while the average for the oldest players is between (3) neither and (2) disagree. If we consider how many hours per week the subjects play computer games, we also notice some differences. For the casual players the mean value was 3.74 , for medium players the average was 3.63 , and for hardcore gamers the mean value was 3.33. This means that the casual players are much more positive to using the mobile phone as a game controller compared to hardcore players. The demographic results on using a mobile phone as a game controller was aligned with our expectations on the outcome. A large percentage of players of games on mobile phones are female and/or young. Hardcore gamers are usually very concerned with and invest money in appropriate game controllers, and thus is not very happy with the shortcomings of using a mobile phone. Although these numbers are not statistically significant, they are strong indicators that align with other player studies. 


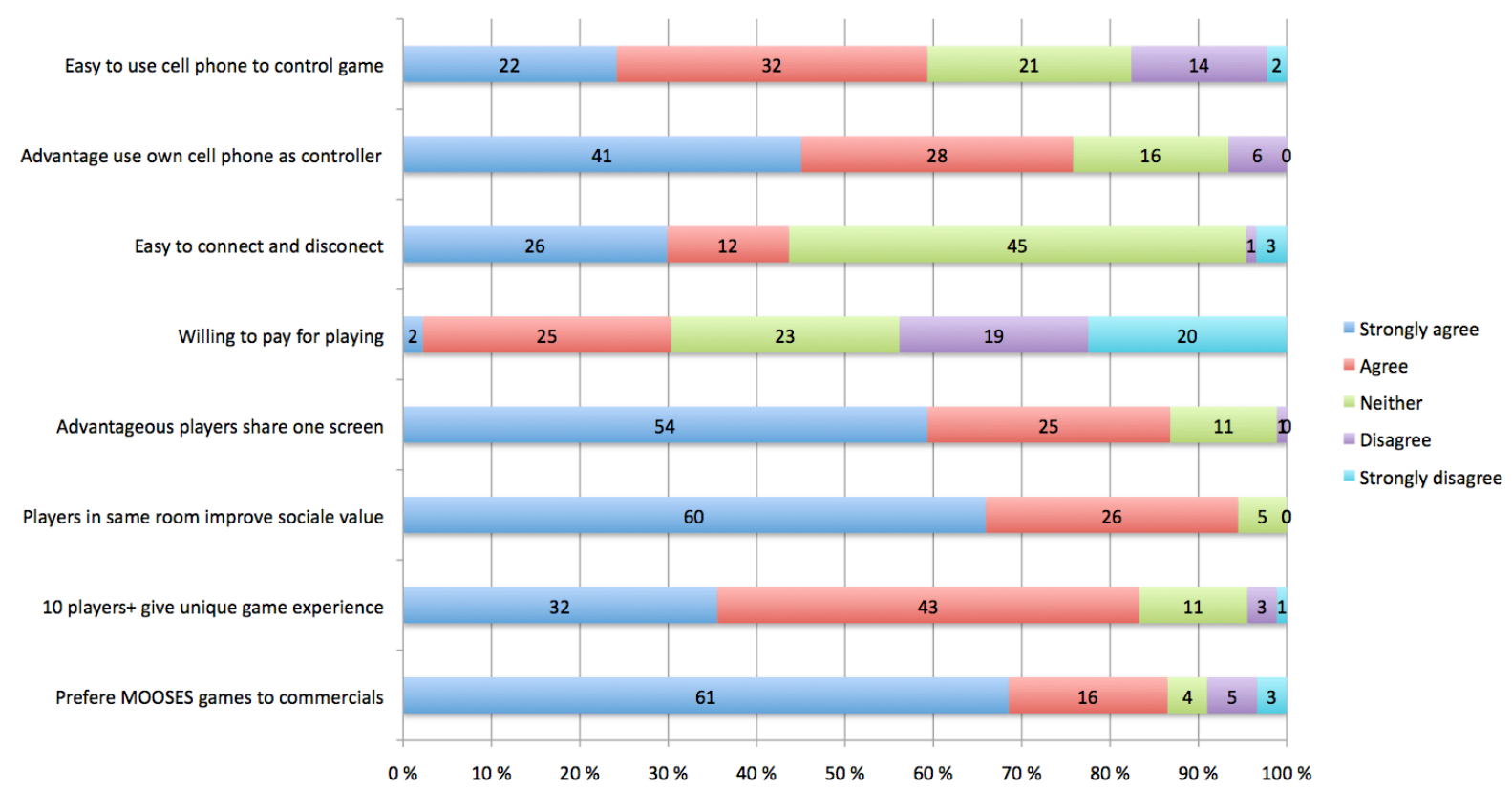

Figure 5. Results from questionnaires on MOOSES games in general

If we consider the statement of the advantage of using own mobile phone as a controller, $76 \%$ were positive, $18 \%$ were neutral, and only $6 \%$ were negative. About the connectivity of MOOSES, $44 \%$ thought it was easy to connect/disconnect, $52 \%$ were neutral, and $4 \%$ thought it was difficult. Further, if we consider the demographics, we find that male subjects in average to a larger degree thought it was easy to connect/disconnect (3.65) compared to female subjects (3.12). There are also similar patterns for age, where the youngest players agreed to a larger degree that connectivity worked well (4.00) compared to the oldest players (3.13). If we compare casual players to hardcore gamers, we find that hardcore gamers to a significantly larger degree found it easy (4.11) compared to casual gamers (3.17).

Regarding the willingness to pay for MOOSES games, $31 \%$ were positive, $26 \%$ were neutral, and $43 \%$ were negative.

If we consider the statements regarding the uniqueness of the MOOSES concept, $87 \%$ were positive that several players shared one large screen, $95 \%$ agreed that players in the same room improved the social value (no subjects disagreed), and finally $75 \%$ agreed that to play more than ten players on one screen gave an unique game experience (12\% neutral). To a large degree, the subjects valued the social aspects and the uniqueness of MOOSES.

In the last statement, the subjects were asked if they preferred a MOOSES commercial game before the movie to a traditional video-based commercial. $87 \%$ of the subjects agreed to this statement, $4 \%$ were neutral, and $9 \%$ disagreed.

The questionnaire included an option for giving a comment on the game. Here are some comments that we received:

- "Cool concept, but it should be possible to use a joystick" (Male, 17-22 years old). MOOSES allow players to use the joystick on the mobile phones, but they are very small and have a much slower response. As the MOOSES concept is built around using a mobile phone as a controller, we cannot change this usability issue. However, by using the accelerometer and multi-touch screens on new smart phones such as iPhone or Android phones, the usability can be improved.

- "It is better to play games, than watch commercials before the movie at the cinemas" (Male 17-22 years old).

- "Fun! I liked the idea to play at the cinema instead of watching commercials :)" (Female 1722 years old)

\subsection{Threats to validity}

The evaluation presented in this paper cannot be classified as a controlled experiment, but rather as user test to assess how users perceive the SelFish game and the MOOSES gaming platform. Still we want to address threats to validity of the evaluation. 
If we consider the internal validity of the evaluation, we can assume that the subjects were randomized as we did not have any control of who volunteered to play the game. We did not have a even representation of subjects in respect to age and sex, but the majority of the subjects were representative to typical gamers (males around twenty years old).

If we consider construct validity, our questionnaire was designed to evaluate both technical and social aspects of MOOSES and SelFish. The questionnaire was designed according to recommended practice using Likert's scale. We also performed a pre-test to check that the procedure for the test and technical set-up worked, and that the questionnaire was clear and easy to comprehend.

Regarding the external validity, we consider the result only valid for our game and platform. We cannot assume that the outcome of this evaluation can be generalized to other evaluation of similar games.

\section{Conclusion}

In this paper, we have presented an unique gaming platform that provide an in-the-same-room gaming experience on a large screen where each player can use his own mobile phone as a game controller. The advantages with this approach is that 1) the player gets two screens (similar to Nintendo DS) where the screen on the mobile phone can be used for individual view of the game, 2) sound, vibration and other features on the mobile phone can be used to give active feedback to the player, and 3) the game installation is simple and low-cost as the players bring their own game controllers.

The paper also described an evaluation of the MOOSES concept and the SelFish game in particular. The evaluation showed that the MOOSES concept worked as planned, but there are some remaining usability issues that must be given extra attention when designing a MOOSES game, such as the keep the controls simple, design the game so it is easy for the player to find his character, and use other means than just the mobile screen to alert the player of important events in the game.

\section{References}

[1] J. McMullan and I. Richardson, "The mobile phone: a hybrid multi-platform medium," in Proceedings of the 3rd Australasian conference on Interactive entertainment Perth, Australia: Murdoch University, 2006.

[2] D. Maynes-Aminzade, R. Pausch, and S. Seitz, "Techniques for Interactive Audience Participation," in
Proceedings of the 4th IEEE International Conference on Multimodal Interfaces: IEEE Computer Society, 2002.

[3] T. Vajk, P. Coulton, W. Bamford, and R. Edwards, "Using a mobile phone as a "Wii-like" controller for playing games on a large public display," Int. J. Comput. Games Technol., vol. 2008, pp. 1-6, 2008.

[4] G. Borriello, C. Hartung, B. Hemingway, K. Koscher, and B. Mayton, "Multi-player soccer and wireless embedded systems," in Proceedings of the 39th SIGCSE technical symposium on Computer science education Portland, OR, USA: ACM, 2008.

[5] S. Boring, M. Jurmu, and A. Butz, "Scroll, tilt or move it: using mobile phones to continuously control pointers on large public displays," in Proceedings of the 21st Annual Conference of the Australian Computer-Human Interaction Special Interest Group: Design: Open 24/7 Melbourne, Australia: ACM, 2009.

[6] J, r. Scheible, T. Ojala, and P. Coulton, "MobiToss: a novel gesture based interface for creating and sharing mobile multimedia art on large public displays," in Proceeding of the 16th ACM international conference on Multimedia Vancouver, British Columbia, Canada: ACM, 2008.

[7] A. S. Shirazi, C. Winkler, and A. Schmidt, "Flashlight interaction: a study on mobile phone interaction techniques with large displays," in Proceedings of the 11th International Conference on Human-Computer Interaction with Mobile Devices and Services Bonn, Germany: ACM, 2009.

[8] V. Pizano, "Volvo game in cinema, URL: http://www.youtube.com/watch?v=cSm5NCbYbCY," 2010. [9] L. Tipping, "Volvo makes waves at the cinema, URL: http://www.mediaweek.co.uk/news/rss/895241/Volvomakes-waves-cinema/," 2009.

[10] M.-L. Rinman, A. Friberg, B. Bendiksen, D. Cirotteau, S. Dahl, I. Kjellmo, B. Mazzarino, A. Camurri, and O. Telenor, "Ghost in the Cave: An Interactive Collaborative Game Using Non-Verbal Communication," in SMAC 03, 2003, pp. 561-563.

[11] Christy, "Cinema Games: MSNBC's NewsBreaker, URL: http://www.christydena.com/2007/06/cinema-gamesmsnbcs-newsbreaker/," 2007.

[12] A. I. Wang, T. Øfsdal, and O. K. Mørch-Storstein, "Lecture Quiz - A Mobile Game Concept for Lectures," in IASTED International Conference on Software Engineering and Application (SEA 2007) Cambridge, MA, USA: Acta Press, 2007, p. 6.

[13] A. I. Wang, T. Øfsdal, and O. K. Mørch-Storstein, "An Evaluation of a Mobile Game Concept for Lectures," in Proceedings of the 2008 21st Conference on Software Engineering Education and Training - Volume 00: IEEE Computer Society, 2008.

[14] Microsoft, "XNA Development Center," http://msdn.microsoft.com/en-us/xnal, March 122009.

[15] H. Korhonen and E. M. I. Koivisto, "Playability heuristics for mobile games," in Proceedings of the 8th conference on Human-computer interaction with mobile devices and services Helsinki, Finland: ACM, 2006. 